\title{
YÜK BARASI ÖLÇÜMLERİNE DAYALI GERİLIMM KARARLILIĞI DEĞERLENDİRMESİ
}

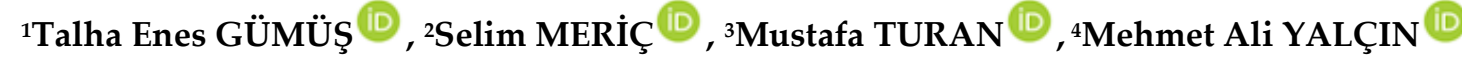 \\ 1,3,4Sakarya Üniversitesi, Mühendislik Fakültesi, Elektrik-Elektronik Mühendisliği Bölümü, Sakarya, TÜRKIYE \\ 2Sakarya Üniversitesi Bilgisayar Araştırma ve Uygulama Merkezi, Sakarya, TÜRKIYE \\ ${ }^{1}$ tgumus@sakarya.edu.tr, ${ }^{2}$ smeric@sakarya.edu.tr, ${ }^{3}$ turan@sakarya.edu.tr, ${ }^{4}$ yalcin@sakarya.edu.tr
}

(Geliş/Received: 01.04.2020; Kabul/Accepted in Revised Form: 08.06.2020)

\begin{abstract}
ÖZ: Bu çalışmada, gerilim kararlılı̆̆ı açısından bir yük barasındaki kritik değerlerin belirlenmesi için, ilgili baraya ait gerilim $(\mathrm{V})$, yük akımı $(\mathrm{I})$ ve güç açışı $(\varphi)$ yerel ölçümlerini kullanarak kestirim yapan iki farklı yaklaşım irdelenmiştir. İlk olarak, yerel bara ölçümlerinden güç sisteminin Thevenin eşdeğer devre parametreleri kestirilmiş, ardından gerilim kararlılığının değerlendirilmesi için gerekli kritik gerilim ve gerilim kararlılık marjini değerleri hesaplanmıştır. Sunulan yaklaşım IEEE 30 baralı test sistemi üzerinde denenmiştir. IEEE 30 baralı standart test sisteminde MATPOWER yazılımı ile güç akışı yapılarak incelenecek baralara ait veriler elde edilmiş ve her iki yöntem ile gerilim kararlılığ gerçeklenmiştir. Benzetim sonuçları, iki yaklaşımında yük baralarındaki gerilim kararlılık sınırlarını başarı ile belirlediği ve baraların gerilim kararlılığı açısından yüklenme düzeylerinin değerlendirilmesinde, kritik baraların tespitinde ve yük atma işlemlerinin önceliklenmesinde kullanılabileceğini göstermiştir.
\end{abstract}

Anahtar Kelimeler: Gerilim kararlılığı, Thevenin eşdeğeri, Gerilim kararlılığı marjini.

\section{Voltage Stability Assessment by Using Local Bus Parameters}

\begin{abstract}
In this study, in order to determine critical values of a load bus with respect to voltage stability, two different estimation approaches using local measurements of voltage (V), current (I) and phase angle $(\varphi)$ on the relevant bus are investigated. First, Thevenin equivalent circuit parameters of the power system is estimated from local bus measurements; then, the critical voltage and voltage stability margin values required for evaluation of the voltage stability are calculated. The proposed approach has been tested on the IEEE 30 bus test system. In IEEE 30 standard test system, the data of the buses to be analyzed was acquired by setting up a power flow with MATPOWER software. The simulation results show that both approaches have successfully determined the voltage stability limits on the load buses and they can be used to evaluate the loading levels of the buses in terms of voltage stability, to determine the critical buses and to prioritize the load shedding operations.
\end{abstract}

Key Words: Voltage stability, Thevenin equivalent, Voltage stability margin.

\section{GİRIŞ (INTRODUCTION)}

Elektrik enerjisi günümüzün en esnek ve en yaygın kullanılan enerji türüdür. Enerji iletim sistemleri tipik olarak; üretim, iletim, dağıtım ve tüketim dizgeleri olarak ele alınmaktadırlar. Günümüzde elektrik şebekeleri çok sayıda ülkeyi de içine alan büyük ölçeklerde enterkonnekte sistemlere dönüşmüş durumdadır. Günümüz alternatif akım şebekelerinde önemli büyüklükler gerilim ve frekans olarak karşımıza çıkmaktadır. Diğer taraftan bakıldığında bu büyük sistem anlık olarak güç arz talebini 
sağlamalı, çeşitli arıza veya bozucu etkilere karşı gerilim ve frekans gibi önemli büyüklükleri kararlı bir şekilde sürdürebilmelidir. Böyle bir sistemin kaçınılmaz olarak kayıpları da vardır. Dolayısı ile beklenti hem kararlı ve hem de ekonomik işletimdir.

Güç sisteminin, olabildiğince verimli ve kabul edilebilir bir çalışma noktasında işletilmesi ve bir bozucuya maruz kaldığında işletme gerilimini istenilen aralıkta tutabilmesi için gerilim kararlılığı değerlendirmelerinin yapılması gerekir.

Bir güç sistemini; diferansiyel, ayrı veya cebirsel olarak modellemek mümkündür. Böyle bir yaklaşım ile kısa veya uzun dönemli analizler yapılabilir. Dinamik etki yapan tüm aktörlerin etkileri izlenebilir [1]. Fakat çok baralı lineer olmayan gerçek bir sisteminin modellenmesi ve doğru verilerin sürdürülmesi çok zaman alıcı olup dinamik benzetimlerle hızlı sonuç alınamamaktadır. Bunun yerine biraz daha sadeleştirilmiş yarı sürekli hal denilebilecek bir yaklaşım ile denklem sayısı azaltılabilir [1,2]. Bu az sayıdaki denklem ile benzetimler yapılarak sistem kararlılığı araştırılabilir.

Gerilim kararlılığı incelemesinde kullanılan en yaygın araç yük akışı analizidir. Bu analizlerde genellikle klasik Newton-Raphson yöntemi kullanılmaktadır [3,4]. Bu hesaplamalarda Jacobian matrisinin tekil olduğu nokta gerilim kararlılık sınırıdır. Yük akışı ile sistemin ilgilenilen barası için P-V ve Q-V eğrileri çizilerek sistemin yüklenme sınırları bulunur. Bu inceleme yaklaşımı sistemlerin planlanma ve yeniden yapılandırılması için daha uygun olmaktadır. Diğer taraftan tekillik noktası civarında NewtonRaphson tabanlı yaklaşımların yakınsama zorluğu bulunduğu da bilinmektedir [5,6].

Gerilim kararlılı̆̆ı incelemesinde kullanılan bir diğer yaklaşım ise " $L$ " indisi diye tanımlanan göstergelerin kullanılmasıdır [7]. Bu indisler hat veya bara yüklenme indisleri olarak da isim almakta olup, pek çok farklı türleri geliştirilmiştir. "L" indisi değerinin 0 olması sistemin yüksüz durumda olduğunu; 1 olması ise hat veya baranın gerilim kararsızlığı oluşturacak şekilde yüklendiğini işaret etmektedir. Bu indisler yük atma içinde kullanılmaktadır [8].

Gerilim kararlılığının değerlendirilmesinde güç sistemin Thevenin eşdeğer empedansına dayalı indisler de oluşturulmuştur $[9,10,11]$. Bu yaklaşım güç sisteminin maksimum güç transferi noktasının belirlenmesine dayanmaktadır. Bu çalışmalarda sorun Thevenin eşdeğer devre parametrelerinin kestirilmesidir. [10]'da en küçük kareler tekniğini içeren bir yaklaşım ve $[11,12,13]$ 'de ters matris çözümüne dayalı bir yaklaşım önerilmiştir. Bu çalışmalarda gerilim kararlılığı sınırlarının belirlenmesi için VSM (Voltage Stability Margine) diye ifade edilen farklı gerilim kararlılık marjinleri tanımlanmıştır. [14]'de farklı L ve VSM göstergeleri ve olası kullanım alanları tartışılmıştır. Son yıllarda yapay zeka tabanlı teknikler kullanılarak VSM hesaplamaları yapılmaktadır[15,16].

Son zamanlarda, fazör ölçüm birimlerin (PMU) geliştirilmesi ile beraber yeni yaklaşımlar geliştirilmeye başlanmıştır. PMU’ların kullanımı ile geniş alan görüntüleme ve kontrolü mümkün olabilecektir. Fakat PMU'lardan sağlanacak gerilim-akım fazör ölçümlerinin mikro saniyeler hassasiyetinde senkronize olması beklenmektedir. Güç sisteminin pek çok barasından senkronize toplanan verilerin bulut bilişim sistemlerinde büyük veri olarak depolanması ve çok hızlı değerlendirilmesi yeni sorunları beraberinde getirmekte olup, bu konuda pek çok çalışma yürütülmektedir. Bu veriler ile anlık gerilim kararlılığı ve diğer değerlendirmelerin yapılması mümkün olabilecektir [17,18,19,20,21,22].

Bu çalışmada öncelikle, gerçek zamanlı gerilim kararlılığı değerlendirmesine yönelik olarak n-baralı bir güç sisteminin incelenecek baradan görünen Thevenin Eşdeğerine indirgenmesi yapilacaktır. Bu amaçla, n-baralı güç sisteminin k. yük barasından yapılan gerilim $(V)$, yük akımı $(I)$ ve güç açışı $(\varphi)$ ölçümlerinden hareketle, k. baradan görülen sistemin Thevenin eşdeğer devre parametrelerinin gerçek zamanlı kestirimine yönelik uygulanan iki farklı yöntem sunularak, elde edilen parametrelerden gerilim kararlılığı değerlendirmesinin nasıl yapılacağı gösterilecek ve elde edilen yaklaşımlar ile IEEE 30 baralı standart test sisteminde yapılan benzetim sonuçları karşılaştırmalı olarak incelenecektir.

\section{GERİLIM KARARLILIĞI PROBLEMİ (VOLTAGE STABILITY PROBLEM)}

IEEE/CIGRE [23] ortak çalışması, gerilim kararlılı̆̆ını; bir güç sisteminin belirli bir çalışma noktasında bir bozucuya maruz kalması sonrasında tüm baralarda gerilimi kabul edilen aralıklarda tutabilme 
yeteneği ve gerilim çökmesini; kabul edilemez aşırı gerilim düşümü, kesintiler veya tüm sistemin devre dışı kalmasına sebep olan olayların oluşturmuş olduğu karmaşık bir süreç olarak tanımlamaktadır.

Güç sistemlerinde kararsızlı̆̆ın sebebi her zaman çok belirgin olmayabilir. Farklı sebepler ile başlayan ve birbirini tetikleyen karmaşık süreçler açı kararsızlığı, frekans kararsızlığı veya gerilim kararsızlığı ile sonlanabilir [13]. Konuya oluş mekanizması veya çözüm önerileri açısından bakıldığında konu daha iyi anlaşılmaktadır. Gerilim kararsızlığı; reaktif güç üretim (arz) sorunları, hat taşıma kapasitesindeki değişimler (paralel hatların açması), seri kompanzasyon sistemlerindeki sorunlar, şönt kompanzasyon sistemlerindeki yetersizlikler, doğrusal olmayan yüklerin davranışları, yük altında kademe değiştirebilen transformatörlerin etkileri, otomatik gerilim regülatörlerinin etkileri, şebeke arızaları sonucunda oluşan aşırı reaktif güç talepleri - düşen transfer kapasitesi gibi etki mekanizmalarının bir veya birkaçının oluşması ile tetiklenebilir. Etki mekanizması reaktif güç tedariki ve gerilim düşümü kaynaklı olduğu için, bu iki unsurun iyi planlanması ve izlenilip önlem alınması ile süreç durdurulabilir. Önlem, hızlı reaktif güç tedariki veya kritik baralardan yük atmak şeklinde alınabilir.

Klasik olarak bir sistem üzerinde gerilim kararlılı̆̆ı, yük akışı yapılıp PV veya QV eğrilerinin burun noktaları araştırılarak değerlendirilir (Şekil 1). Fakat bu yaklaşım tüm sistem parametrelerinin bilinmesini gerektirdiğinden, gerçek zamanlı değerlendirmeler için uygun değildir.

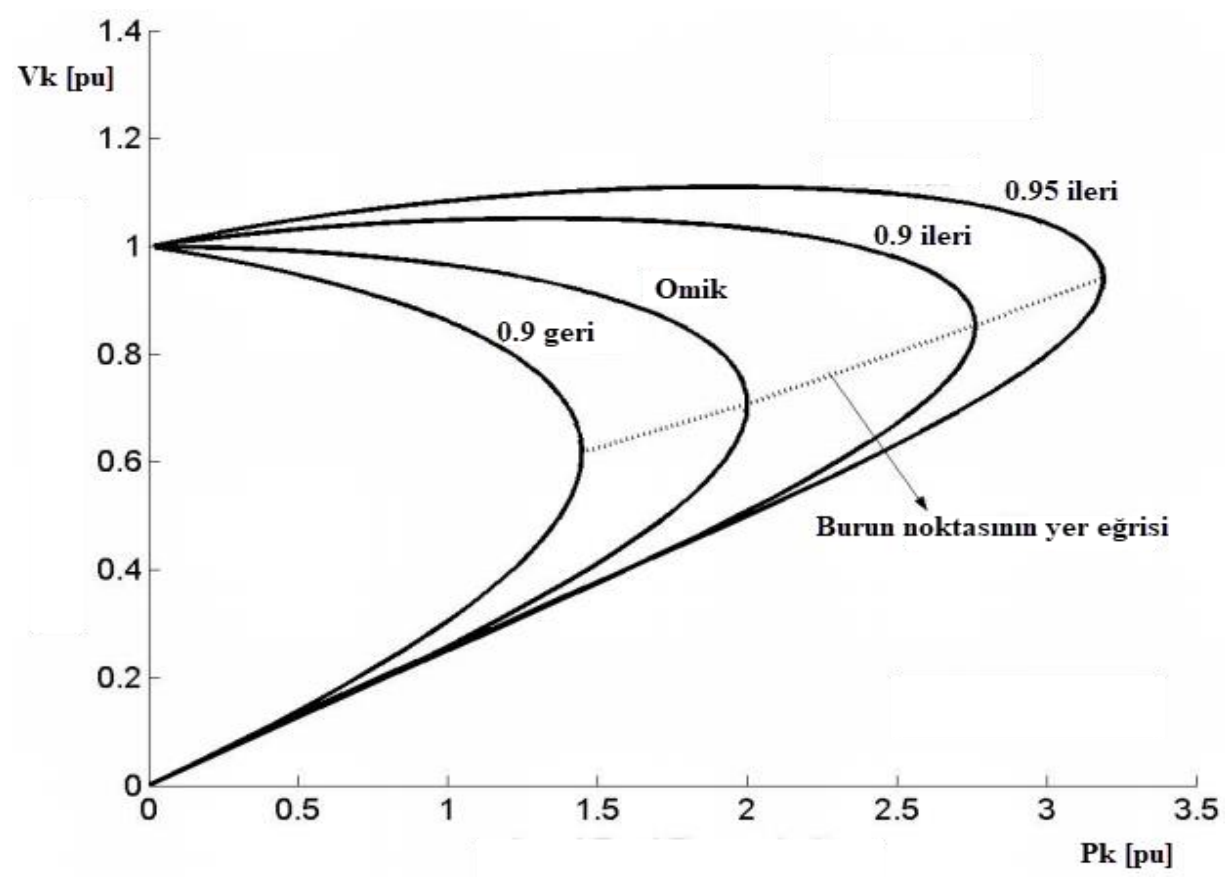

Şekil 1. k. yük barasında farklı güç faktörlü yüklenmeler için çizilmiş tipik bir P-V eğrisi.

Figure 1. A typical P-V curve plotted for different power factor loads at the $k$. load bus. 


\section{THEVENİN EŞDEĞER DEVRE PARAMETRELERİ KESTIRIMM YÖNTEMİ (ESTIMATION METHOD OF THEVENIN'S EQUIVALENT CIRCUIT PARAMETERS)}

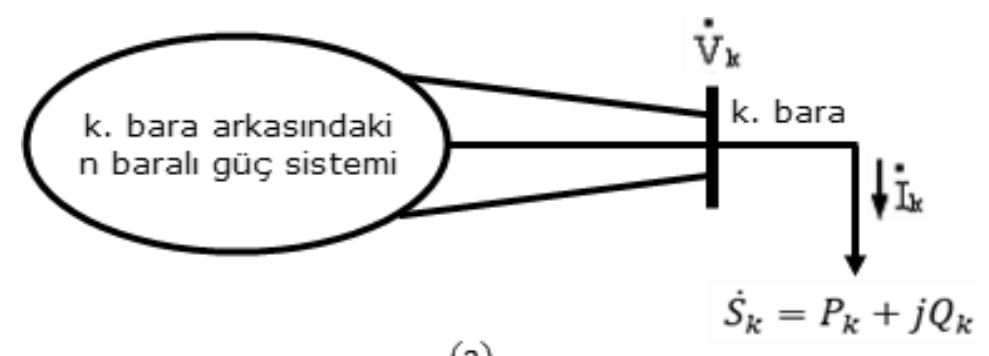

(a)

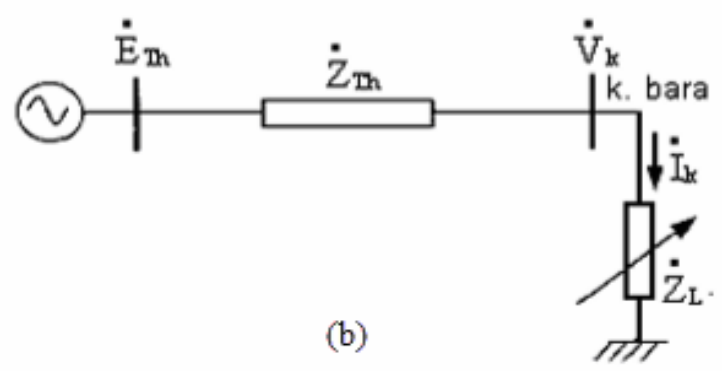

Şekil 2. (a) k. bara arkasında kalan güç sistemi, (b) Thevenin Eşdeğeri ve yük Figure 2. (a) The power system behind $k^{\text {th }}$ bus. (b) Thevenin Equivalent and load.

Şekil 2'de n-baralı bir sistemin k. yük barasından bakıldığında görünen Thevenin eşdeğerini temsil etmektedir. $V_{k}$ referans alınarak bu devre için Kirchoff çevre eşitliği;

$E_{T h} \cdot e^{j \delta}=V_{k}+I_{k} \cdot e^{j \varphi_{k}} \cdot\left(R_{T h}+j X_{T h}\right)$

yazılabilir. Bu eşitlik gerçel eksen $\alpha$, ve imajiner eksen $\beta$ olmak üzere iki eşitlik takımı düzenlenerek yazılırsa;

$E_{T h} \cdot e^{j \delta}=V_{k}+I_{k} \cdot e^{j \varphi_{k}} \cdot\left(R_{T h}+j X_{T h}\right)$

$E_{\beta}-I_{k} \cdot R_{T h} \cdot \operatorname{Sin}\left(\varphi_{k}\right)-I_{k} \cdot X_{T h} \cdot \operatorname{Cos}\left(\varphi_{k}\right)=0$

yazılabilir. Bu iki eşitlik $\mathrm{E}_{\alpha}, \mathrm{E}_{\beta}$, $\mathrm{R}_{\mathrm{Th}}$ ve $\mathrm{X}_{\mathrm{Th}}$ 'dan oluşan dört bilinmeyeni (Thevenin eşdeğer parametreleri) çözümlemek için yeterli değildir. Bunun için k. baradan ardıl en az iki ölçüm alınmalıdır [11]. Bu ölçümler k. baradan ardıl olarak sürekli alınacaktır. İlk ölçüm 1, ikinci ölçüm 2 indisi ile gösterilmek üzere;

$\left[\begin{array}{rrrr}-I_{1} \operatorname{Cos}\left(\varphi_{1}\right) & I_{1} \operatorname{Sin}\left(\varphi_{1}\right) & 1 & 0 \\ -I_{2} \operatorname{Cos}\left(\varphi_{2}\right) & I_{2} \operatorname{Sin}\left(\varphi_{2}\right) & 1 & 0 \\ -I_{1} \operatorname{Sin}\left(\varphi_{1}\right) & -I_{1} \operatorname{Cos}\left(\varphi_{1}\right) & 0 & 1 \\ -I_{2} \operatorname{Sin}\left(\varphi_{2}\right) & -I_{2} \operatorname{Cos}\left(\varphi_{2}\right) & 0 & 1\end{array}\right] \cdot\left[\begin{array}{c}R_{T h} \\ X_{T h} \\ E_{\alpha} \\ E_{\beta}\end{array}\right] \cdot\left[\begin{array}{c}V_{1} \\ V_{2} \\ 0 \\ 0\end{array}\right]$ 


$$
A=\left[\begin{array}{rrrr}
-I_{1} \operatorname{Cos}\left(\varphi_{1}\right) & I_{1} \operatorname{Sin}\left(\varphi_{1}\right) & 1 & 0 \\
-I_{2} \operatorname{Cos}\left(\varphi_{2}\right) & I_{2} \operatorname{Sin}\left(\varphi_{2}\right) & 1 & 0 \\
-I_{1} \operatorname{Sin}\left(\varphi_{1}\right) & -I_{1} \operatorname{Cos}\left(\varphi_{1}\right) & 0 & 1 \\
-I_{2} \operatorname{Sin}\left(\varphi_{2}\right) & -I_{2} \operatorname{Cos}\left(\varphi_{2}\right) & 0 & 1
\end{array}\right] \quad x=\left[\begin{array}{c}
R_{T h} \\
X_{T h} \\
E_{\alpha} \\
E_{\beta}
\end{array}\right] \quad b=\left[\begin{array}{c}
V_{1} \\
V_{2} \\
0 \\
0
\end{array}\right]
$$

Tanımlanarak, ters matris yöntemi ile bilinmeyen vektörü çözülebilir:

$A \cdot x=b \quad ; \quad x=A^{-1} \cdot b$

Literatür ile karşılaştırıldığında, elde edilen bu çözüm, Haque' nin [11] çözümünün sadeleştirilmiş haline denk gelmektedir(Yöntem 1). Bu sadeleştirme, ters matris işleminde oluşan tekillik sorununda iyileşme sağlamaktadır. $6 \mathrm{~b}$ eşitliğinin çözümünden, sistemin Thevenin eşdeğer geriliminin etkin değeri, yük açısı, eşdeğer empedans ve empedans açısı;

$$
\begin{aligned}
& E_{T h}=\sqrt{E_{\alpha}^{2}+E_{\beta}^{2}} ; \delta=\operatorname{atan}\left(\frac{E_{\alpha}}{E_{\beta}}\right) \\
& Z_{T h}=\sqrt{R_{T h}^{2}+X_{T h}^{2}} ; \theta=\operatorname{atan}\left(\frac{X_{T h}}{R_{T h}}\right)
\end{aligned}
$$

elde edilir. V referans seçildiğinden, $7 \mathrm{~b}$ ifadesinde bulunan yük açısı, k. baraya (yüke) doğru olan beslemelerde pozitif çıkacaktır.

Yapılan çeşitli benzetimlerde, 7a ifadesinin Thevenin gerilimini \%1'den küçük hatalar ile kestirdiği görülmüştür. Matris çözümünden (Denklem 5) elde edilen $E_{T h}$ ile $Z_{T h}$ için yan çözümler geliştirilmiştir [13] (Yöntem 2). Bir tek $E_{T h}$ 'nin bile yüksek doğrulukta bulunması, sürece büyük katkı yapmaktadır. Zira $Z_{T h}$ için yan çözümler geliştirilebilmektedir.

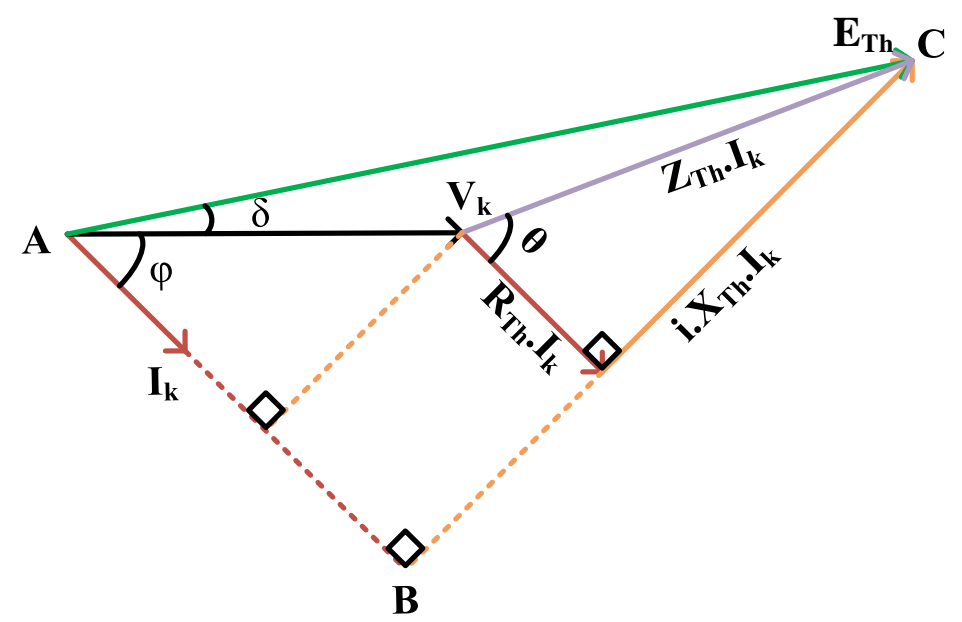

Şekil 3. Eşitlik 1 içim çizilmiş fazör diyagram

Figure 3. Phasor diagram drawn for equation 1

Kestirimler yapılırken, temel kabullerden ilki k. baradan yapılan ölçüm çiftleri esnasında $E_{T h}{ }^{\prime \prime n}$ sabit kaldığıdır. Fazör diyagram üzerinde tanımlanan $\mathrm{ABC}$ üçgeni için;

$E_{T h}^{2}=\left(V_{k} \cdot \operatorname{Cos}\left(\varphi_{k}\right)+I_{k} \cdot Z_{T h} \cdot \operatorname{Cos}(\theta)\right)^{2}+\left(V_{k} \cdot \operatorname{Sin}\left(\varphi_{k}\right)+I_{k} \cdot Z_{T h} \cdot \operatorname{Sin}(\theta)\right)^{2}$ 
ile verilen Pisagor bağıntısı yazılabilir. Bu aşamada $Z_{T h}$ ve $\theta$ haricindeki tüm parametreler bilinmektedir. Orta gerilim ve yüksek gerilim hatları için $\theta$ açısı 70-85 derece aralığında çıkmaktadır [13]. Bu durum, bu çalışmada yapılan benzetim çalışmaları sürecinde de gözlenmiştir. Ayrıca çeşitli yük ve işletme şartlarında $Z_{T h}$ ve $\theta^{\prime}$ nın çok az değiştiği gözlenmiştir. Çalışmanın devamında $\theta$ sabit ve ortalama 80 derece alınacaktır. $\theta ; Z_{T h}$ 'in hesaplanmasına çok ciddi etki yapmamaktadır. Zira bu çalışmada sadece $Z_{T h}$ 'in genliği ile ilgilenilmekte ve yapılacak gerilim kararlılığı değerlendirmesi için $R_{T h}$ ve $X_{T h}{ }^{\prime} 1 n$ ayrı ayrı bulunması gerekmemektedir. Yapılan benzetimlerde $\theta$ 'daki 10 derecelik bir hatanın empedansta maksimum \%4 hata getirdiği, devamında yapılan hesaplamalarda hatanın artmadığı gözlenmiştir.

9 Nolu eşitlikten $Z_{T h}$ 'nin kökleri çözülüp, pozitif yanıt üreten kök alınırsa;

$Z_{T h}=\frac{\sqrt{4 \cdot E_{T h}^{2}-2 \cdot V_{k}^{2}+2 \cdot V_{k}^{2} \cdot \operatorname{Cos}\left(2 \varphi_{k}-2 \theta\right)}-2 \cdot V_{k} \cdot \operatorname{Cos}\left(\varphi_{k}-\theta\right)}{2 \cdot I_{k}}$

bulunur. Yine Şekil 3'ten $\delta$ açısı hesaplanırsa;

$\delta=\operatorname{acos}\left(\frac{E_{T h}^{2}-I_{k}^{2} \cdot z_{T h}^{2}+V_{k}^{2}}{2 \cdot E_{T h} \cdot V_{k}}\right)$

olarak bulunur. Böylece, $7 \mathrm{a}$ ve 10 numaralı eşitlikler ile $\mathrm{n}$ baralı güç sisteminin Thevenin eşdeğer parametreleri, k. baradan yapılan V, I ve $\varphi$ ölçümleri kullanılarak kestirilmiş olur.

Bir sistemin Thevenin eşdeğer devresinin bilinmesi, yaygın bilinen maksimum güç transferi ilkelerinin uygulanması ile, o sistemden transfer edilebilecek maksimum güç sınırı hakkında kesin bilgi sağlar.

\section{GERILIMM KARARLILIĞININ DEĞERLENDİRILMESİ (EVALUATION OF VOLTAGE STABILITY)}

Güç sistemlerinde kararlılık değerlendirmesi yapılacak barada gerilim kararlılığı sınırlarının değerlendirilebilmesi için, ilgili baradan çekilebilecek maksimum gücün bilinmesi gerekir. Yaygın olarak bilinen maksimum güç teoremine göre, Şekil $2 b^{\prime}$ deki sistemin yüke transfer edebileceği maksimum gücün, her bir faz için;

$\left|Z_{Y \ddot{u} k}\right|=\left|Z_{T h}\right|$

Yük empedansının, güç sisteminin ilgili barasından bakıldığında görülen Thevenin eşdeğer devre empedansına eşit olması durumunda gerçekleştir. Bu durum gerilim kararlılığı açısından k. bara için sınır (kritik) değerdir. Buradan hareketle, kritik durumda;

$$
\left|Z_{Y \ddot{k} k}\right| \cdot I_{k}=\left|Z_{T h}\right| \cdot I_{k}
$$

olacaktır. Maksimum güç transferi için çevre denklemleri yazılırsa;

$$
\begin{aligned}
& \dot{E}_{T h}=\dot{Z}_{T h} \cdot \dot{I}_{Y u ̈ k}+\dot{Z}_{Y \ddot{u} k} \cdot \dot{I}_{Y \ddot{u} k} \\
& \dot{E}_{T h}=\dot{I}_{Y \ddot{u} k} \cdot\left(2 \cdot \dot{Z}_{T h}\right)
\end{aligned}
$$

denklemleri elde edilir. Sistemden maksimum güç transfer edildiğinden ilgili baradan çekilen akım kritik akım olacaktır.

$\dot{I}_{\text {Kritik }}=\frac{\dot{E}_{T h}}{2 \cdot \dot{z}_{T h}}$ 
İlgili baradan kritik akım çekildiği esnada (maksimum güç transferi) baraya ait fazör diyagramı Şekil $4^{\prime}$ de verildiği gibi olacaktır.

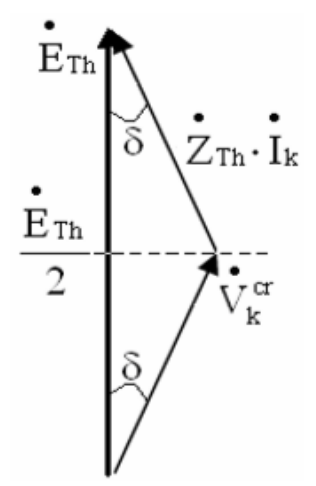

Şekil 4. Kritik durumda gerilim fazörleri

Figure 4. Voltage phasors in critical condition

Şekil 4'den gerilim kararlılığ 1 değerlendirmesi yapılacak bara için kritik gerilim değeri ve gerilim kararlılık marjini $\left(V S M_{V}\right)$;

$V_{k \text { ritik }}=\frac{E_{T h}}{2 \operatorname{Cos} \delta}, V_{k \text { ritik }} \geq \frac{E_{T h}}{2}$

$V S M_{V}=\frac{V_{\text {ölçülen }}-V_{k \text { ritik }}}{V_{k r i t i k}}$

olarak tanımlanır [13]. Burada $V S M_{V} ; 1$ ile 0 arasında değer almalıdır. $V S M_{V}$ 'nin 1 olması, test barasının yüksüz olduğunu gösterir; bu değer sıfıra yaklaşması, baranın taşıma kapasitesinin sınırına gelindiğini, diğer bir deyişle gerilim kararsızlığına girileceğini ifade eder.

Bu çalışmada ilk olarak Denklem 5'in çözümünden elde edilen $E_{T h}$ ile Denklem 10'dan elde edilen $Z_{T h}$ değerleri için ilgili baraya ait $V_{k \text { ritik }}$ ve $V S M_{V}$ marjini kritik noktaya ulaşana kadar adım adım hesaplanarak kararlılık analizi yapılmıştır (Yöntem 1). Daha sonra sadece Denklem 5'in çözümünden elde edilen $E_{T h}$ ve $Z_{T h}$ değerleri kullanılarak ilgili baranın $V_{k \text { ritik }}$ ve $V S M_{V}$ marjini ilgili baralar için tekrar adım adım hesaplanmış (Yöntem 2) ve her iki yöntem ile elde edilen sonuçların karşılaştırılması yapılmıştır. 


\section{BENZETIM ÇALIŞMASI (SIMULATION STUDY)}

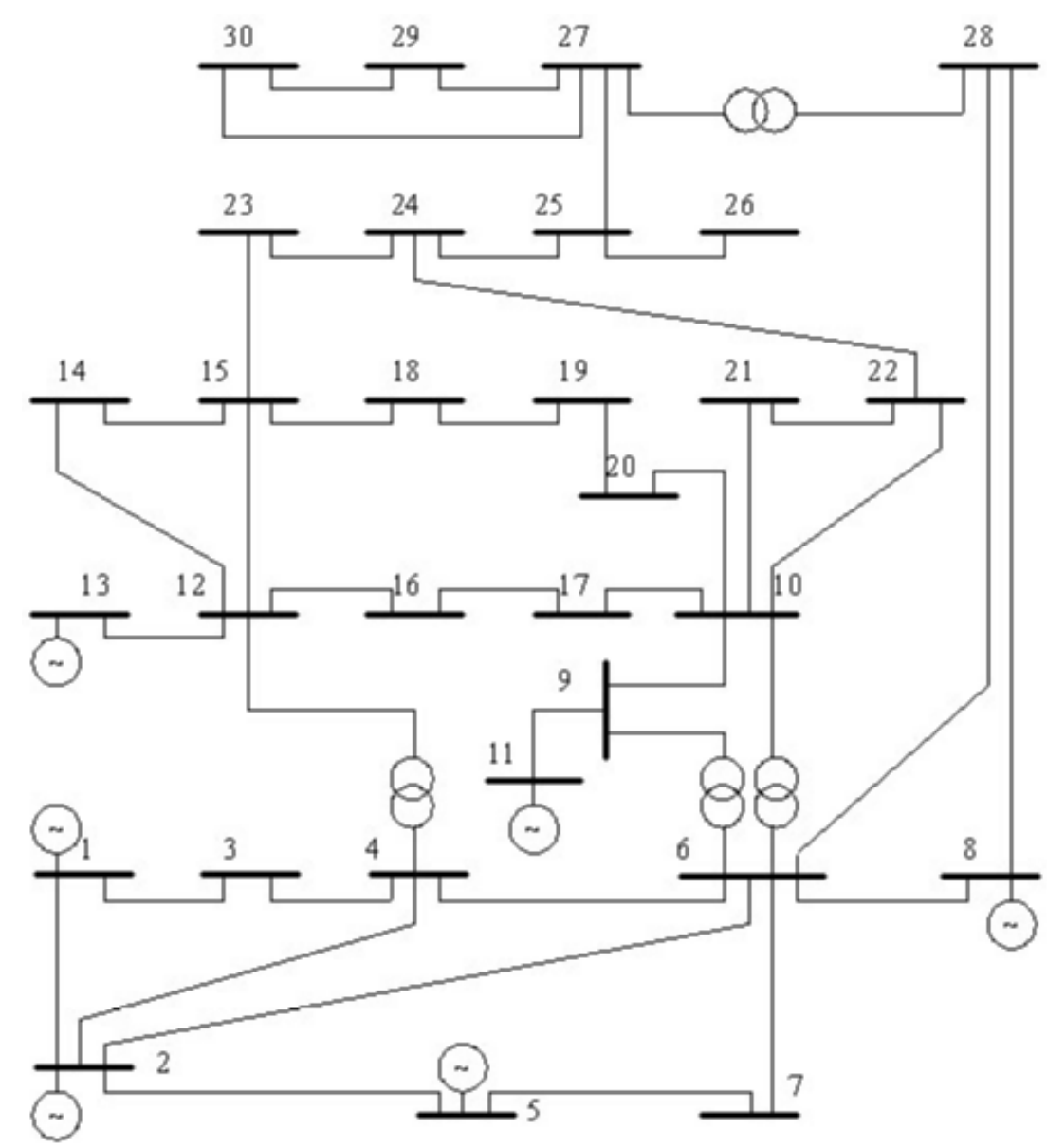

Şekil 5. IEEE30 baralı standart test sistemin tek hat şeması [21]

Figure 5. Single line diagram of IEEE30 bus standard test system

Yukarıda anlatılan her iki yöntem Şekil 5'te verilen IEEE 30 baralı standart test sisteminin 7, 14, 19 ve 30 numaralı yük baralarında denenmiş ve her baraya ait sonuçlar karşılaştırılmalı olarak verilmiştir. Grafiklerde ilk olarak baraya ait kritik gerilim değeri ve bara gerilim değeri her bir yüklenme değeri için sistem kritik noktaya ulaşana kadar adım adım hesaplanarak her iki yöntem için verilmiştir. Kritik gerilim ve bara gerilimi birbirine eşit olduğunda sistemin kritik değere ulaştığı anlaşılmaktadır (Şekil 6,8,10,12). Daha sonra kritik gerilim değerleri kullanılarak hesaplanan $V S M_{V}$ marjinleri her iki yöntem için karşılaştırmalı olarak ilgili baralardaki sonuçları verilmiştir (Şekil 7,9,11,13). Burada marjin değeri sıfır olduğunda sistemin kritik değerlere ulaştığı anlaşılmaktadır. Bütün şekillerde sisteme ait gerçek kritik noktalar da dikey çizgi olarak verilmiştir. 


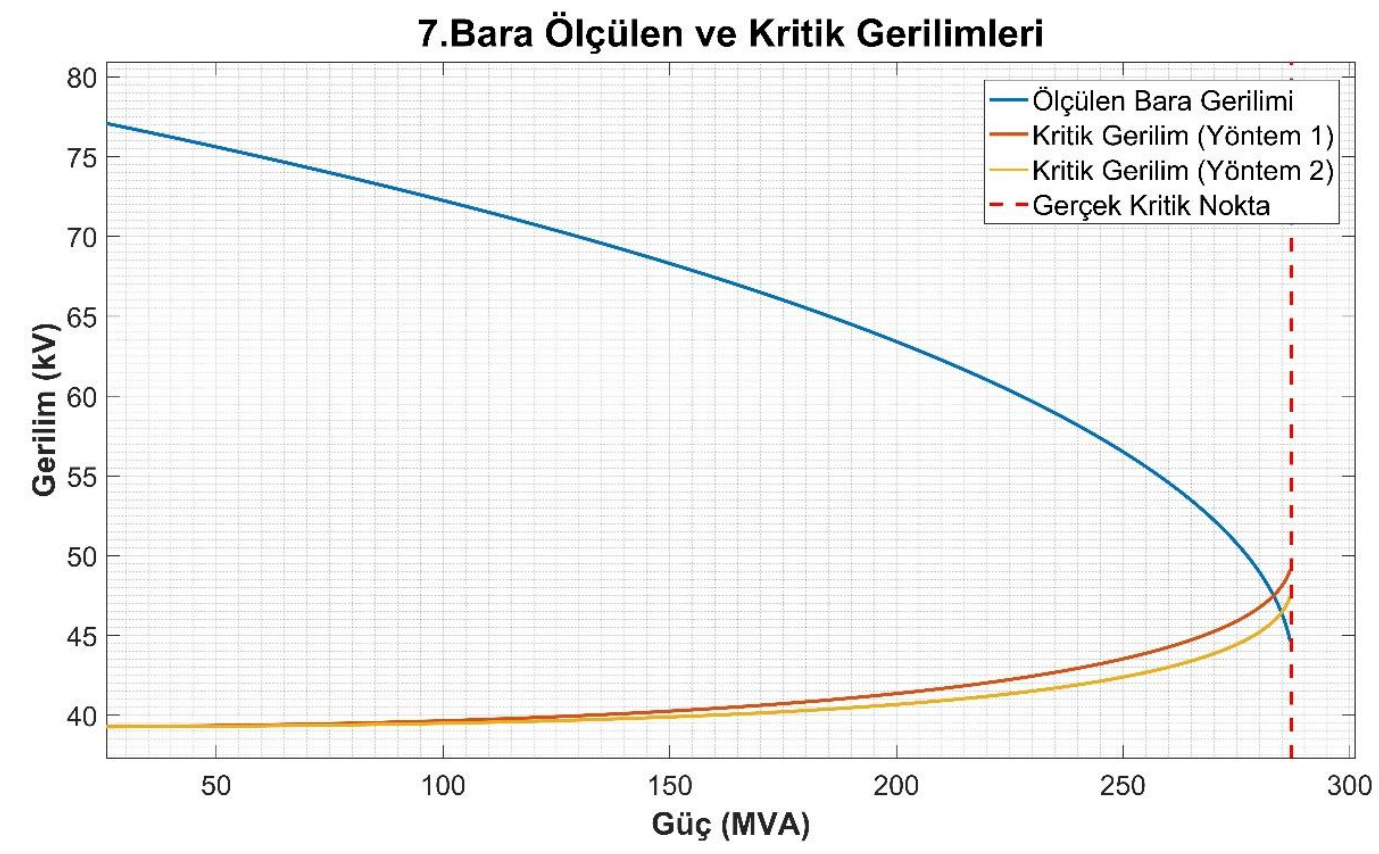

Şekil 6. Bara 7 için ölçülen ve kritik gerilimler grafiği Figure 6. Measured and critical voltages graph for Bus 7

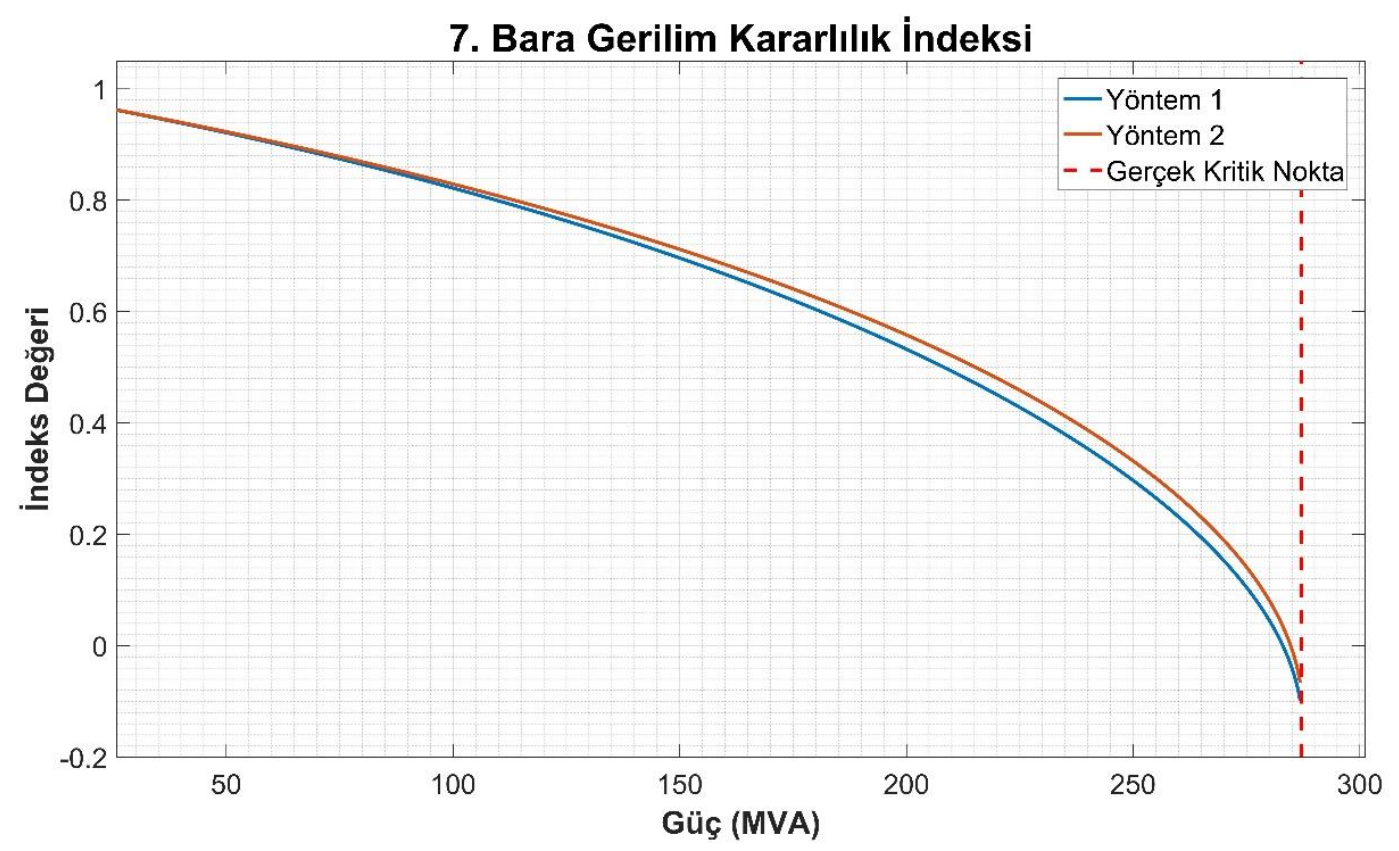

Şekil 7. Bara 7 için gerilim kararlılık marjini Figure 7. Voltage stability margin for Bus 7 


\section{Bara Ölçülen ve Kritik Gerilimleri}

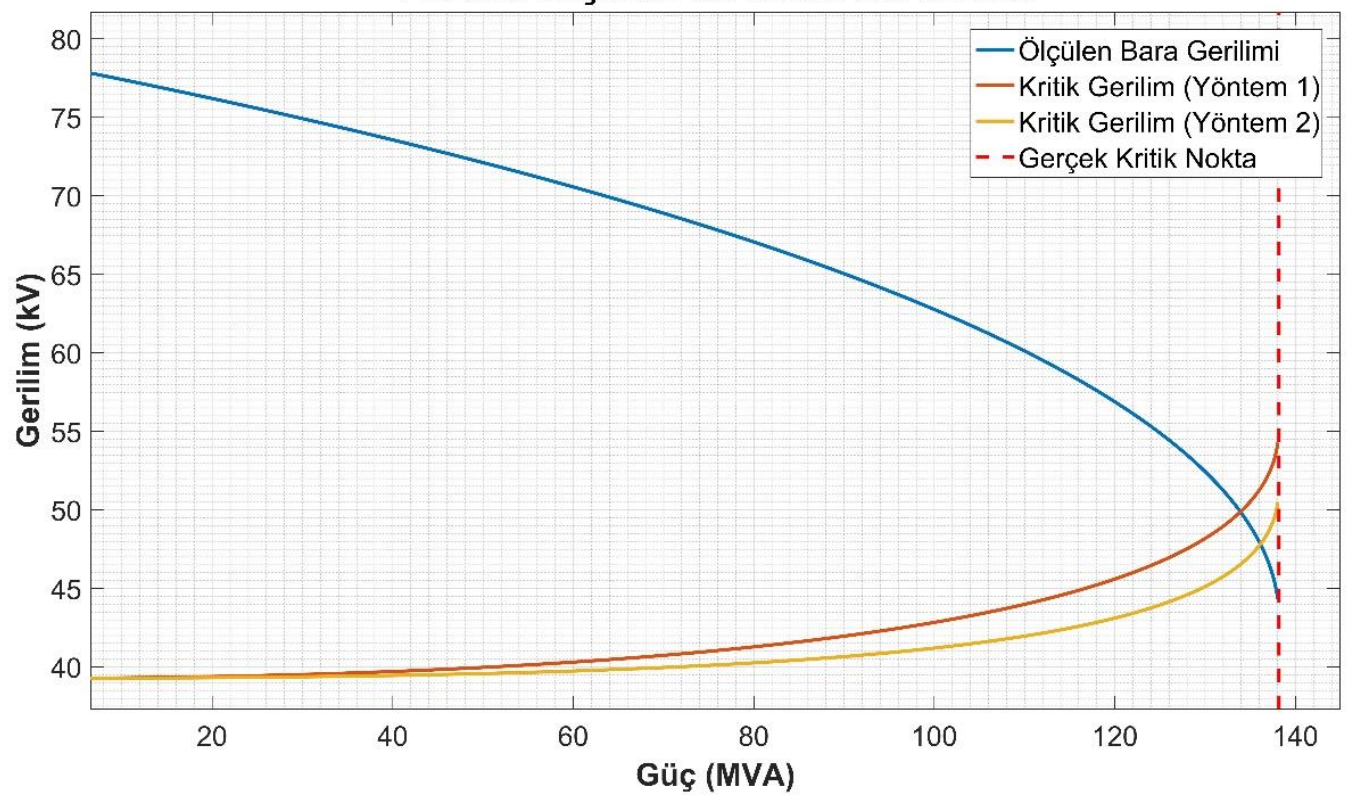

Şekil 8. Bara 14 için ölçülen ve kritik gerilimler grafiği

Figure 8. Measured and critical voltages graph for Bus 14

14. Bara Gerilim Kararlılık İndeksi

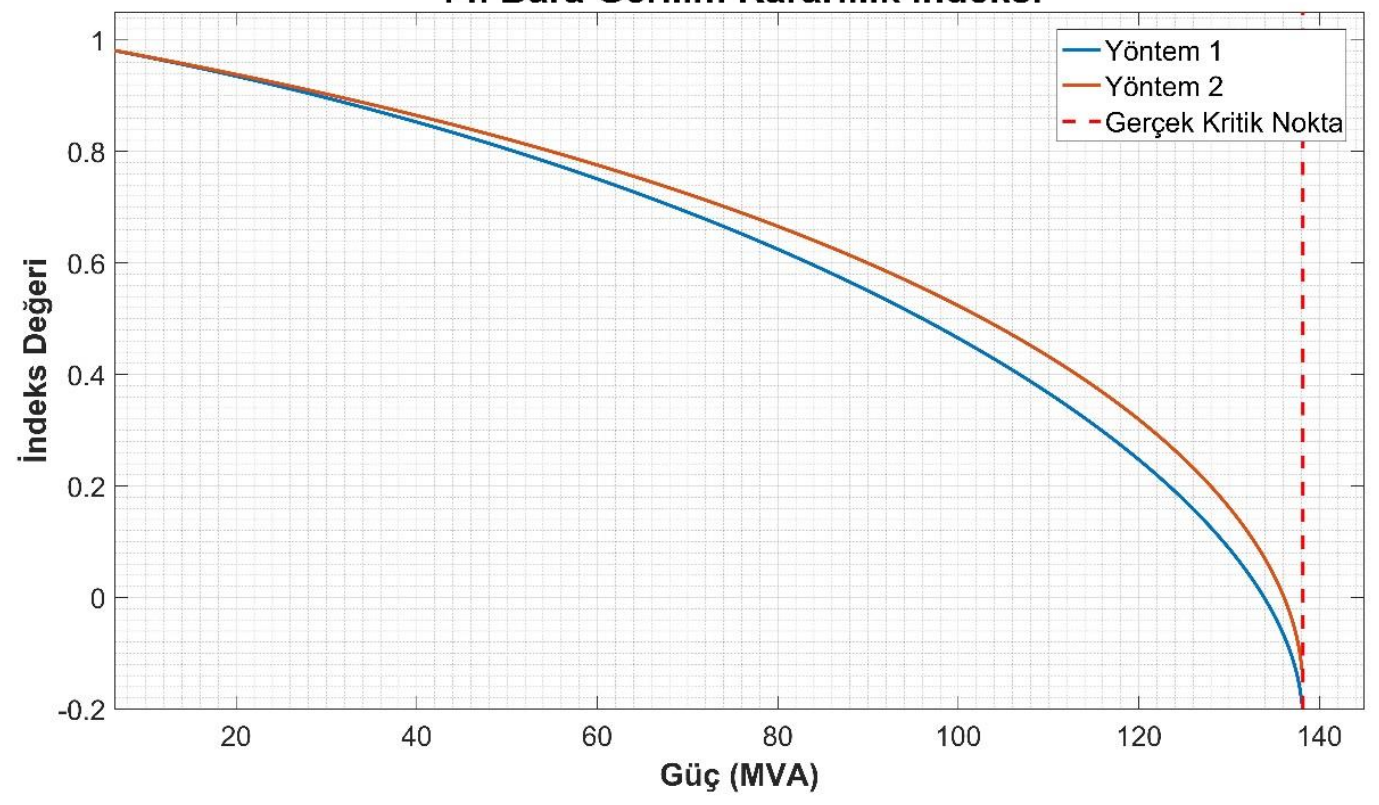

Şekil 9. Bara 14 için gerilim kararlılık marjini

Figure 9. Voltage stability margin for Bus 14 
19. Bara Gerilim Kararlılık İndeksi

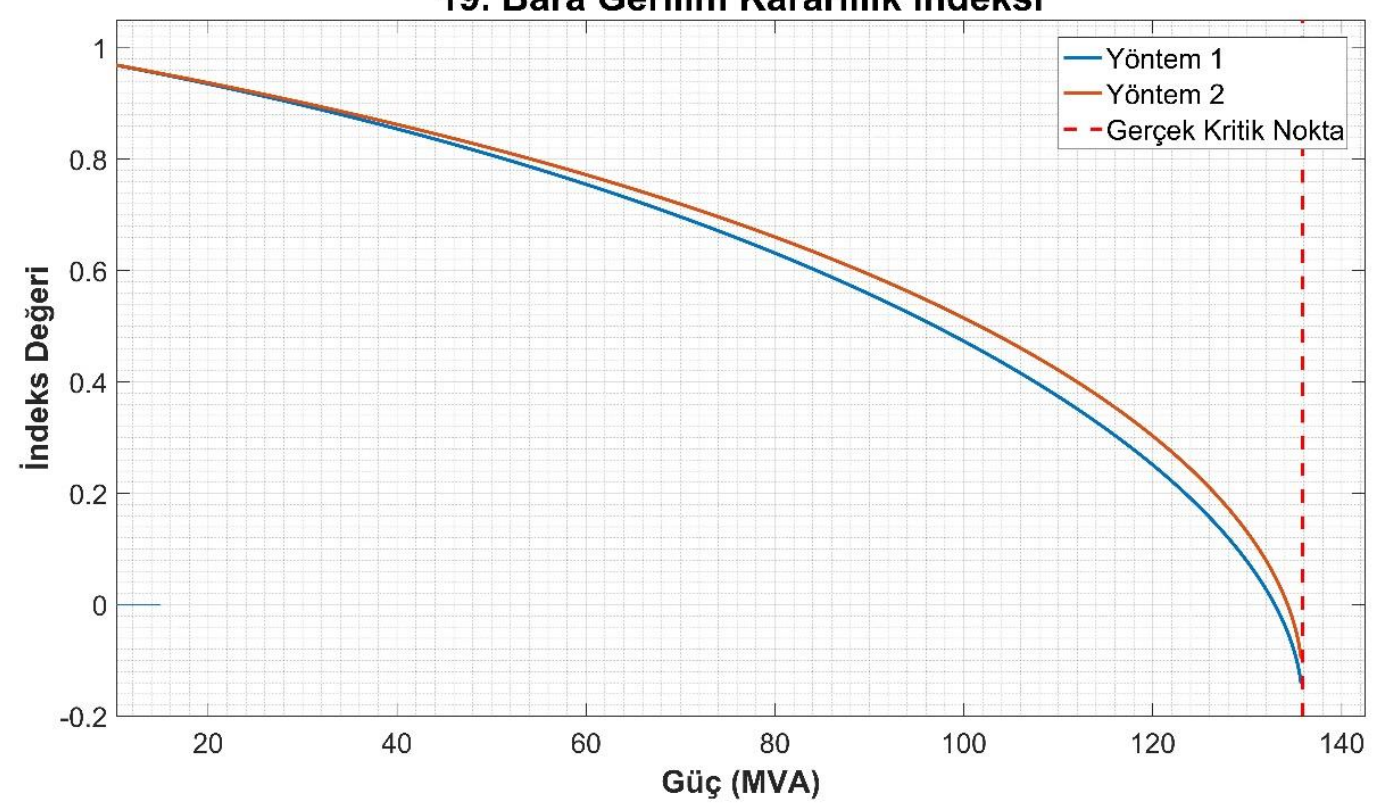

Şekil 10. Bara 19 için ölçülen ve kritik gerilimleri grafiği

Figure10. Measured and critical voltages graph for Bus 19

19. Bara Gerilim Kararlılık İndeksi

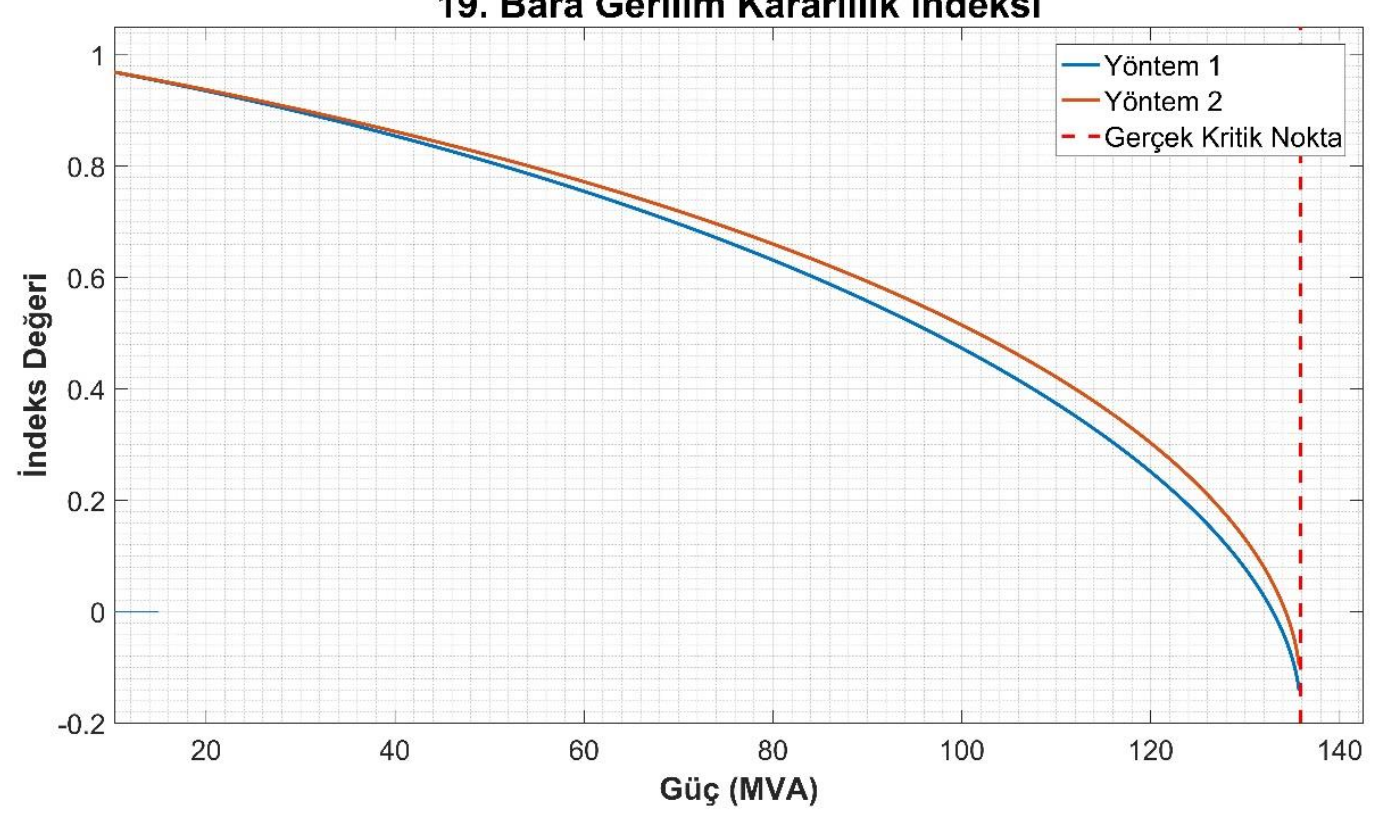

Şekil 11. Bara 19 için gerilim kararlılık marjini

Figure 11. Voltage stability margin for Bus 19 


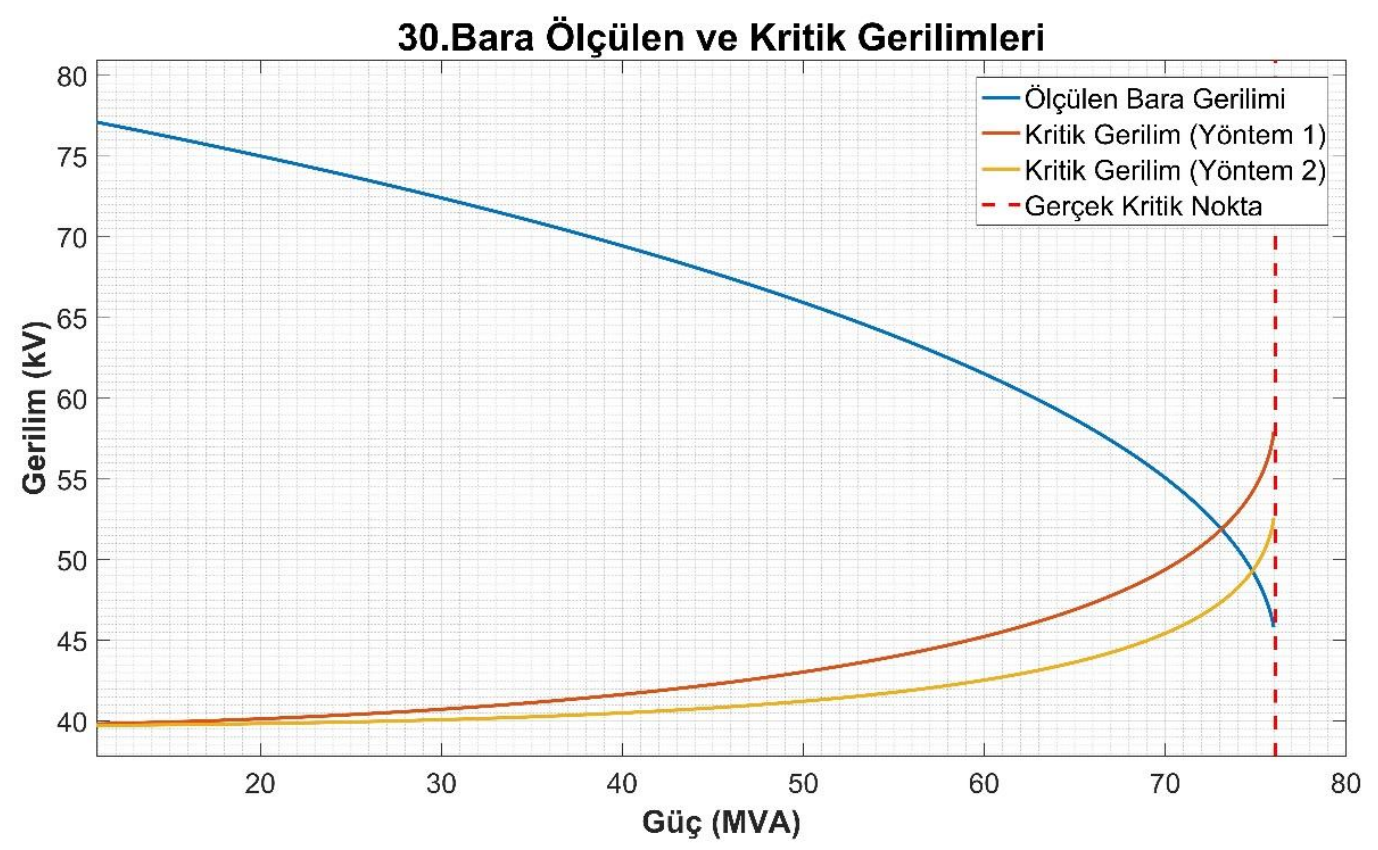

Şekil 12. Bara 30 için ölçülen ve kritik gerilimleri grafiği

Figure 12. Measured and critical voltages graph for Bus 30

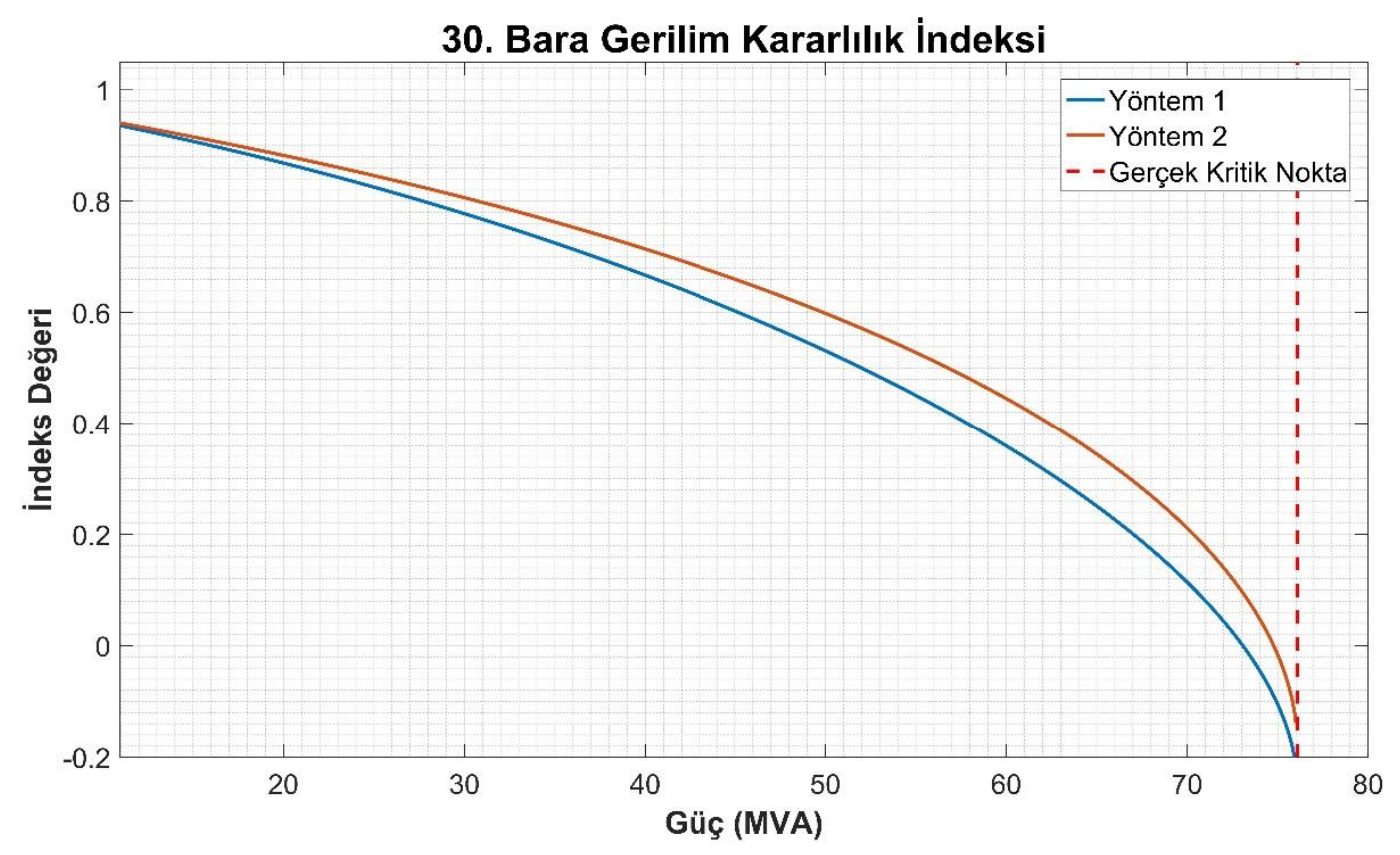

Şekil 13. Bara 30 için gerilim kararlılık marjini

Figure 13. Voltage stability margin for Bus 30

IEEE 30 baralı Test sisteminin 7, 14, 19 ve 30 numaralı yük baralarından elde edilen veriler kestirim algoritmasında işlenerek analizi yapılacak olan baralara ait kritik gerilimler ve gerilim kararlığı marjinleri $\left(V S M_{V}\right)$ hesaplanmıştır. Tüm testlerde gerilim kararlılı̆̆ sınırı doğru olarak belirlenmiş olup 7, 14, 19 ve 30 numaralı baralara ait kritik gerilim ve bara gerilimi grafikleri Şekil 6, 8,10 ve 12' de her iki yöntem için verilmiştir. Analizi yapılan baralara ait $V S M_{V}$ marjinleri Şekil 7, 9,11 ve 13'de verilmiştir. $V S M_{V}$ sıfır olduğu $S$ gücü, analizi yapılan baraları gerilim kararsızlı̆̆ına sokan kritik güçlerdir. ( $V S M_{V}$ negatif değer almaz, Şekil 7, 9, 11 ve 13'deki negatif değerler, Yöntem 1'de kararsızlık noktasının gerçek kararsızlık noktasına göre erken kestirilmiş olmasından kaynaklanmıştır). 


\section{SONUÇLAR (CONCLUSIONS)}

Bu çalışmada iki farklı yaklaşım ile IEEE 30 baralı test sisteminin 7, 14, 19 ve 30 numaralı yük baralarında gerilim kararlılığı analizi yapılmıştır. Yük baralarına ait V, I ve $\varphi$ değerleri MATPOWER yazılımı ile bara yükleri adım adım arttırılıp güç akışı yapılarak elde edilmiş ve bu verilere dayalı olarak gerilim kararlılı̆̆ 1 analizi yapılmıştır. Elde edilen verilerle ilk olarak analizi yapılacak baraya ait $E_{T h}$ ve $Z_{T h}$ değerleri Yöntem 1 ile hesaplanmıştır. Hesaplanan $E_{T h}$ ve $Z_{T h}$ değerleri ile Denklem 17 'de verilen formül ile baralara ait $V_{k r i t i k}$ değerleri hesaplanmıştır. Daha sonra Yöntem 2 ile analizi yapılan baralara ait $E_{T h}$ ve $Z_{T h}$ değerleri hesaplanmış ve hesaplanan bu değerler ile baralara ait $V_{k r i t i k}$ değerleri hesaplanmıştır.

Elde edilen verilerle analizi yapılacak baraya ait $E_{T h}$ ve $Z_{T h}$ değerleri iki farklı Yöntem ile ayrı ayrı hesaplanmıştır. Hesaplanan $E_{T h}$ ve $Z_{T h}$ değerleri kullanılarak incelenen baralara ait kritik gerilim $\left(V_{k r i t i k}\right)$ değerleri belirlenmiştir.

$V_{\text {kritik }}$ değerlerinin yük akışı ile adım adım hesaplanan (veya ölçülen) bara gerilimine eşit olduğu (kesiştiği) noktada sistemin kritik değerlerine ulaşacağı öngörülmektedir. Ancak, her iki yöntemle de hesaplanan kritik noktalarının, ilgili baranın gerçek kritik noktasından daha küçük değerde kaldığı görülmektedir.

Hesaplanan $V_{\text {kritik }}$ değerleri Denklem $18^{\prime}$ te verilen gerilim kararlılığ marjini ile değerlendirilmiştir. Yöntem 1 ve Yöntem 2 ile elde edilen sonuçların hepsinde $V S M_{V}{ }^{\prime}$ nin gerilim kararsızlık sınırlarını maksimum \% 5 hata sınırı içerisinde kestirdiği görülmüştür. Her iki yöntem ile elde edilen $V S M_{V}$ marjinleri gerilim kararlılığı bakışı ile yük baralarının yüklenilebilme sınırı hakkında yeterli doğrulukta bilgi üretmektedir.

Yöntem 2'de, Yöntem 1'e göre daha az işlem yapılmaktadır. Buna rağmen, yapılan analizlerde Yöntem 2 ile belirlenen kritik gerilim ve kritik güç noktalarının gerçek kritik değerlere daha yakın olduğu görülmüştür.

Yöntem 1 ve Yöntem 2 ile sadece yük barasına ait veriler kullanılarak sistemin kritik değerlere yakınlığı hakkında bilgi edinilerek, güç sistemlerinin gerilim kararsızlığına girmesini engellemek için ilgili baradaki yükün atılması veya diğer önlemlerin alınması için kullanılabilir.

\section{KAYNAKLAR (REFERENCES)}

Van Cutsem, T., Vournas, C.,1998, "Voltage Stability of Electric Power Systems", Kluwer Academic Publishers.

Van Cutsem, T., Vournas, C.,1996, "Voltage Stability Analysis in Transient and Mid-term Time Scales," IEEE Transactions in Power Systems, Cilt 11, Sayı 1, ss. 146-154,

Glover, J.D., Sarma, M.S.,2001, "Power System Analysis and Design", Brooks/Cole, 3. Bask1,

Crow, M., 2002, "Computational Methods for Electric Power Systems", CRC Yayınları.

Canizares, C.A., De Souza, A.Z., Quintana, V.H., 1996, "Comparison of Performance Indices for Detection of Proximity to Voltage Collapse," IEEE Transactions on Power Apparatus and Systems, Cilt 11, Say1 3, ss. 1441-1450.

Chiang, H.D., Jean-Jumeau, R.,1993, "Toward a Practical Performance Index for Predicting Voltage Collapse in Electric Power Systems", Proceeding of the 1993 IEEE/PES Summer Meeting.

Kessel, P., Glavitsch, H.,1986," Estimating The Voltage Stability of A Power System, IEEE Transactions on Power Systems", Cilt 1, Sayı 3, ss. 346-354, 
Tuan, T.Q., Fandino, J., Hadjsaid, N.J., Sabonnadiere, C., VU., H.,1994,“'Emergency Load Shedding to Avoid Risks of Voltage Instability Using Indicators", IEEE Transactions on Power Systems, Cilt 9, Sayı 1, ss. 341-351.

Vu, K., Begovic, M., Novosel, D., Saha, M.,1999,'”Use of Local Measurements to Estimate Voltage-Stability Margin", IEEE Transactions on Power Systems, Cilt 14, Say1 3, ss. 1029-1035.

Milosevic, B. , Begovic, M.,2003,'”Voltage-Stability Protection and Control Using a Wide-Area Network of Phasor Measurement", IEEE Transactions on Power Systems, Cilt 18, Sayı 1, ss. 121-127.

Haque, M.H. ,2003," On-line Monitoring of Maximum Permissible Loading of A Power Within The Voltage Stability Limits", IEEE Proceedings of Generation Transmission and Distribution, London, UK.

Turan, M; Demircioğlu, S.B. ; Yalçın, M. A. ,2006," Voltage Stability Evaluation by Using Maximum Power Transfer Phasor Diagram", Journal of Applied Sciences, Cilt 6,Say1 13,ss. 2809-2812, Asian Network for Scientific Information

Demircioğlu, S. B. ,2006, “Enerji Sistemlerinde Gerilim Kararlılığ Sintrlarının Yerel Bara Parametreleri Kullanılarak Gerçek Zamanlı Değerlendirilmesi", Doktora Tezi, Sakarya Üniversitesi, Fen Bilimleri Enstitüsü, Elektrik-Elektronik Mühendisliği Bölümü.

Cardet, C.E.D, Analysis on Voltage Stability Indices, AACHEN University, Master Thesis, 2010.

Ghaghishpour A., Koochaki A.,2020," An intelligent method for online voltage stability margin assessment using optimized ANFIS and associated rules technique" ISA Transactions.

Rodriguez-Garcia L.,Perez-Londono S., Mora-Florez J.,2019," An optimization-based approach for load modelling dependent voltage stability analysis,", Electric Power Systems Research,, Cilt 177, ss. 105960.

Glavic, M., Van Cutsem T.,2009," Wide-Area Detection of Voltage Instability from Synchronized Phasor Measurements", Part I: Principle, IEEE Transactions on Power Systems, Cilt 24, Sayı 3, ss. 1408-1416.

Glavic, M., Van Cutsem T.,2009,'”Wide-Area Detection of Voltage Instability from Synchronized Phasor Measurements", Part II: Simulation Results, IEEE Transactions on Power Systems, Cilt 24, Sayı 3, ss. 1417-1425.

Glavic, M., Van Cutsem T.,2008," Detecting with PMUs The Onset of Voltage Instability Caused by A Large Disturbance", Proceedings 2008, IEEE Power Engineering Society General Meeting, Pittsburgh, PA.

Burchett, Stephen M.,Douglas, Daniel,Ghiocel, Scott G.,2018," An Optimal Thévenin Equivalent Estimation Method and its Application to the Voltage Stability Analysis of a Wind Hub", IEEE Transactions on Power Systems ,Cilt 33, Say1 4, ss. 3644-3652.

A. Mahari, H. Seyedi, 2016," A wide area synchrophasor-based load shedding scheme to prevent voltage collapse", International Journal of Electrical Power and Energy Systems, Cilt 33, ss. 248-257.

IEEE/CIGRE Joint Task Force on Stability Terms and Definitions,2004, "Definition and Classification of Power System Stability", IEEE Transactions on Power Systems, Cilt 19, Sayı 2, ss.1387-1401.

Mandoulidis P., Vournas C.,2020," A PMU-based real-time estimation of voltage stability and margin", Electric Power Systems Research, Cilt 178, ss. 106008.

IEEE 30 Bus Standart Test System, https://www.al-roomi.org/power-flow/30-bus-system, (10.05.2019). 